\title{
Evaluation of Power Minimization for Routing Protocol and Multicast Networks
}

\author{
Shabir Ahmed Mir'1, T. Padma² \\ ${ }^{1}$ Research scholar, Information Technology, AMET University, Chennai \\ ${ }^{2}$ Department of computer Applicaions, Sona College of Technology, Salem
}

\begin{tabular}{l}
\hline Article Info \\
\hline Article history: \\
Received Oct 6, 2017 \\
Revised Dec 12, 2017 \\
Accepted Jan 3, 2018 \\
\hline
\end{tabular}

Keywords:

\begin{abstract}
In this paper explore the scenario of wireless multicast with a single source and multiple relay that together convey consecutively refined sources to multiple receiver. Joint source-channel coding (JSCC) protocol is proposed aimed at mitigating the complexity and difficulty in signal synchronization in multicast taking end-to-end mean cube error deformation as the quality of service measure the problems of JSCC communication are formulated to minimize the total power utilization where both relay selection and power allocation at the base station (BS) and all the relays are jointly determined to solve the formulated problem that is nonetheless nonlinear in nature a twostep iterative algorithm is investigated for power allocations at all transmitters where a in order quadratic programming method is developed into find a strict local minimum. To reduce the computation complexity a heuristic algorithm for relay selection is presented. Case studies are conducted to verify the proposed formulations and solution methods.
\end{abstract}

Copyright (c) 2018 Institute of Advanced Engineering and Science. All rights reserved.

\section{Corresponding Author:}

Shabir Ahmed Mir,

Research scholar, Information Technology,

AMET University,

Chennai.

\section{INTRODUCTION}

Due to nature of wireless channels, wireless communication suffers from multipath fading and the time-varying characteristic that causes distortion to the delivered information. This has imposed a stringent limitation on some applications where retransmission is not possible, such as broadcast/multicast to resolve the problem Joint source channel coding (JSCC) and cooperative transmissions are two classes of solutions that exploit different design dimensions of communication systems.

The advent of wireless mobile ad-hoc networks (MANETs) has offered an efficient and most importantly cost effective technique to make use of the availability of mobile hosts when no fixed infrastructure is provided. In MANET the mobile nodes can easily communicate with each other while they are freely moving around in different directions.

An ad-hoc network relies entirely on nodes cooperation for forwarding information from data sources to intended destination nodes. Some examples of mobile nodes in an ad-hoc network are laptop computers, smart phones and personal digital assistants that interact directly with each other. There are many advantages of such an ad-hoc network which include fast deployment, robustness, efficiency and natural support for mobility. Genetic algorithms based enhanced K Strange points clustering algorithm is also describes that [1]. Media Access Delay and Throughput Analysis of Voice Codec with Silence Suppression on Wireless Ad Hoc Network explained in [2]. An Heterogeneous Population-Based Genetic Algorithm for Data Clustering is described here. The optimal clusters are achieved by modifying the genetic algorithm [3]. 


\section{PROPOSED SYSTEM}

Introduce an asynchronous multi-relay aided two-slot JSCC multicast protocol that can deal with successively refined sources of arbitrary numbers of layers, in which the optimal DFE receiver and the optimal SINR level of each layer in the relayed phase are jointly considered.

Formulate a suite of optimization problems by taking total energy usage as the target, where the transmit power levels at the BS and relays as well as the power ratio of each layer are jointly determined.

Develop a sequential quadratic program through power parameters optimization and a heuristic algorithm to optimally select relays in transmission to further minimize the total power consumption to reduce the calculation complication a heuristic algorithm for communicate selection is obtainable.

\subsection{Architecture of JSCC}

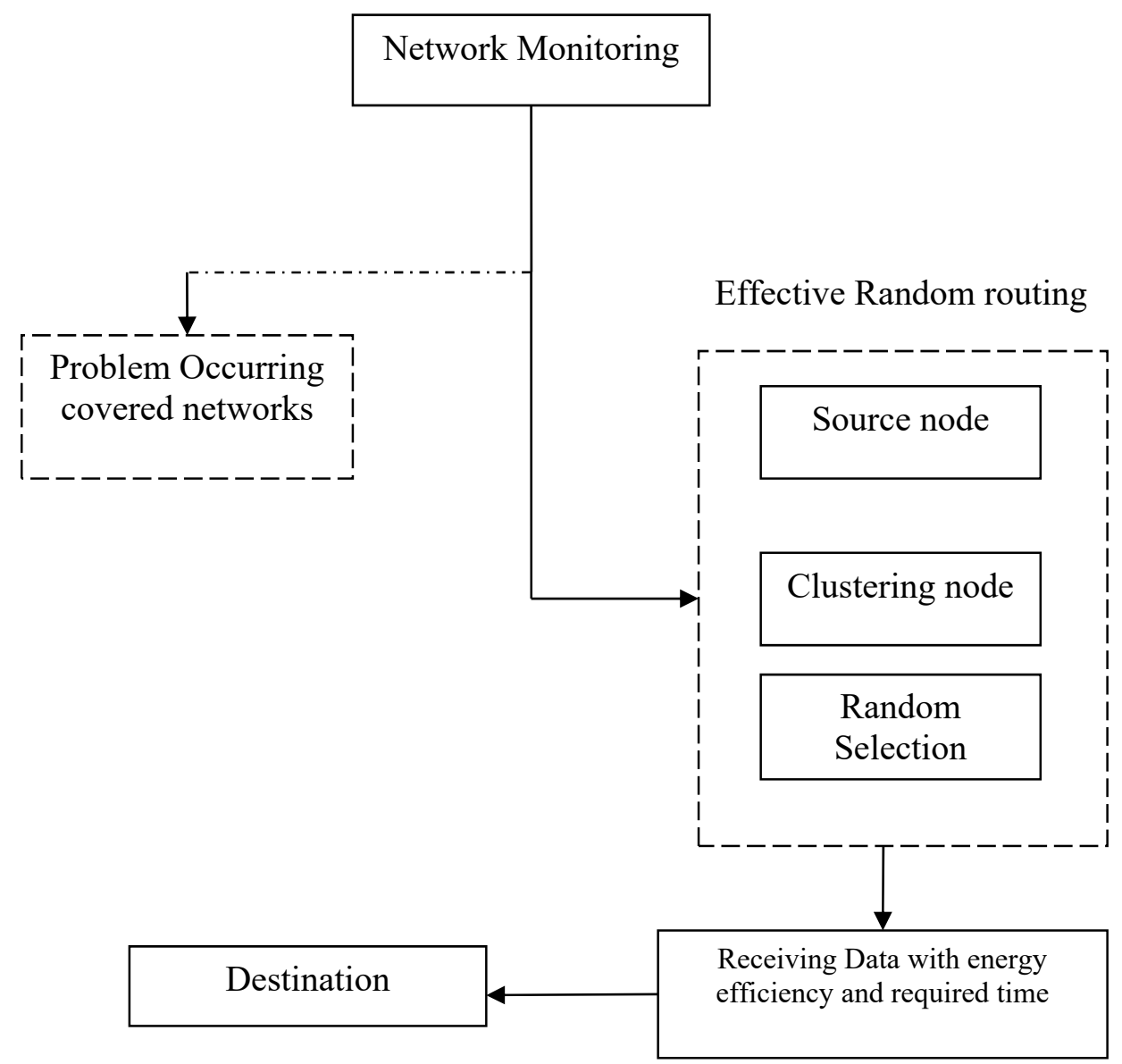

\section{DISCUSSION}

Asynchronous multi-relay aided two-slot JSCC multicast protocol is proposed and their lifetime is calculated. Optimization problems is formulated by taking total energy usage as the target, where the transmit power levels at the BS and relays as well as the power ratio of each layer are jointly determined. Multi-relay aided two-slot JSCC multicast protocol is obtained and the results are compared with their conventional method. Figure 1 describes the network lifetime for both the proposed and existing schemes. The proposed method has better network lifetime compared to the existing one. 


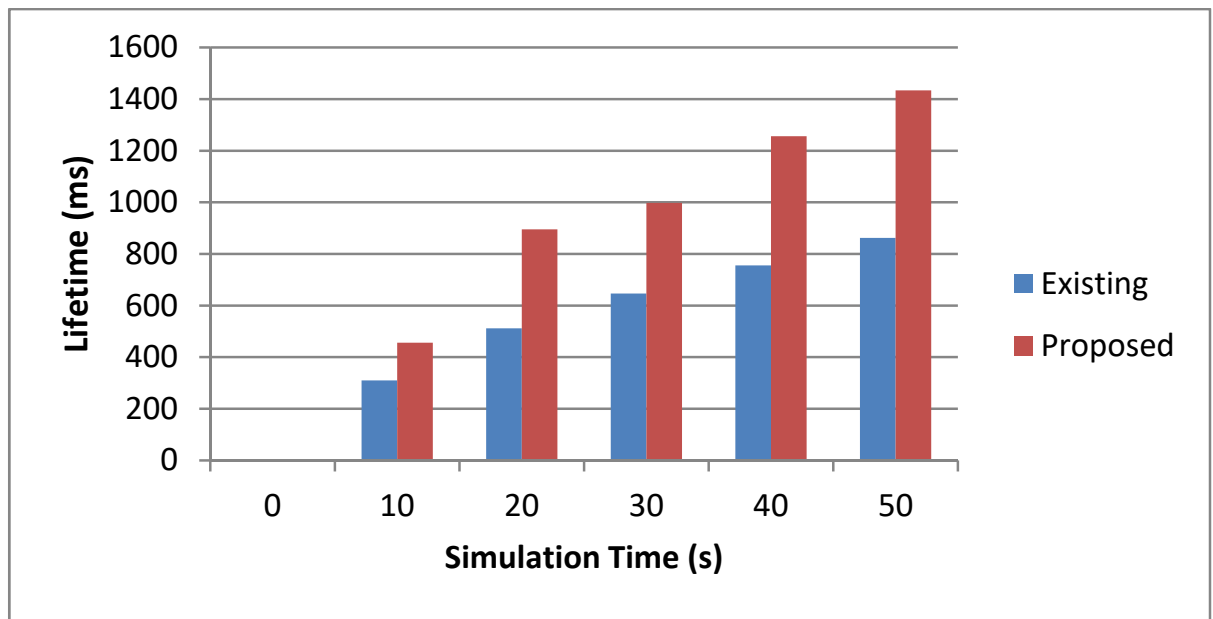

Figure 1. Network Lifetime

\section{CONCLUSION}

The paper considered a multi-relay aided joint source-channel coding (JSCC) multicast network containing a source multiple decode-and-forward relays and multiple destination nodes. Due to the difficulty in synchronizing the relay communication an asynchronous JSCC two-slot multicast protocol was introduced along with an optimal DFE structure where the associated SINR of all layers can be determined based on MMSE criterion. EED at the end nodes was taken as the performance metric for the system optimization subject to the power level and power ratio assigned to each layer at the transmitters. An optimization problem to minimize total influence utilization was formulate and solved where the transmit power level power assigning ratio for all layers, and relay selection can be jointly strong-minded.

\section{REFERENCES}

[1] Johnson T. and Singh S. K., "Genetic algorithms based enhanced K Strange points clustering algorithm," in Computing and Network Communications (CoCoNet), International IEEE Conference, pp. 737-741, 2015.

[2] Shah R. D. and Singh S. K., "Media Access Delay and Throughput Analysis of Voice Codec with Silence Suppression on Wireless Ad Hoc Network," Procedia Computer Science, vol. 79, pp. 940-947, 2016.

[3] A. Bedboudi, et al., "An Heterogeneous Population-Based Genetic Algorithm for Data Clustering," Indonesian Journal of Electrical Engineering and Informatics (IJEEI), vol/issue: 5(3), 2017. 